\title{
La Arqueología medieval en España: antecedentes y estado actual
}

\author{
Ricardo Izquierdo Benito*
}

\section{ANTECEDENTES}

Hasta épocas todavía recientes -frente a otras etapas históricas que habían experimentado un importante desarrollo en sus investigaciones arqueológicas (la Prehistoria y la Edad Antigua)- la Arqueología Medieval no había despertado mucho interés en nuestro país. Las causas de esta situación son difíciles de explicar, aunque en ello pudieron influir algunos factores que seguidamente analizaremos.

En primer lugar, la consideración de que la Arqueología es una disciplina a aplicar para la reconstrucción de aquellos periodos históricos que no han generado una documentación escrita o en los que ésta es muy escasa. Éste sería, por tanto, el caso de la Edad Media, de la que se conserva, en general, un repertorio documental abundante, que es el que, de preferencia, cuando no con exclusividad, han utilizado los historiadores en sus investigaciones. De ahí el poco interés -y a veces hasta el desprecio- que los medievalistas han concedido tradicionalmente a esta disciplina, lo que ha supuesto que se haya considerado a la Arqueología como "en las afueras del medievalismo", en afortunada expresión de Miquel Barceló (1988).

Por otra parte, la labor arqueológica, en algunos momentos, no ha estado exenta de un cierto interés por la búsqueda de objetos valiosos, en el sentido más material del término. La Edad Media, por las especiales circunstancias del desenvolvimiento del quehacer humano en aquella época, no posibilitó la producción de piezas de gran valor -salvo casos muy excepcionales y con un destino social muy concre- to- susceptibles de ser recuperadas por vía de una excavación. De aquí también, por tanto, que ello incidiese en un cierto desinterés por esta época para algunos arqueólogos, sabedores de que no iban a encontrar objetos muy valiosos al excavar un yacimiento medieval.

Tampoco hay que olvidar los derroteros historiográficos por los que ha discurrido el análisis de la Edad Media, especialmente en algunos planteamientos teóricos -el feudalismo, por ejemplo- en los que parecía que, en principio, la Arqueología poco podía decir. Los aspectos de carácter más cotidiano (vivienda, ajuares domésticos, etc.) que son los que, de una forma más directa, los resultados de una excavación pueden informar, no eran entonces tenidos muy en consideración.

Y además, en el caso concreto de nuestro país, no habría que dejar de lado la posible incidencia que la trayectoria histórica de la Península Ibérica pudo haber tenido en este sentido, ya que, frente a la evolución más o menos "lineal" que otras naciones europeas experimentaron a lo largo de la Edad Media, el fraccionamiento político que aquí se produjo, desembocó en la constitución de áreas geopolíticas diferenciadas, que presentaban sus propias peculiaridades arqueológicas. Ello, por consiguiente, dotaba a la Edad Media peninsular de una mayor complejidad arqueológica y exiǵa una mayor preparación para el arqueólogo que pretendiese abordarla en su conjunto.

Todos estos factores, unidos a otros que, seguramente, se nos escapan, consideramos que deben ser tenidos en cuenta a la hora de intentar com- 
prender el escaso desarrollo que, hasta épocas no muy lejanas, la Arqueología Medieval había tenido en España. Todo ello quedaba reflejado en una escasa producción bibliográfica relativa a los temas de Arqueología Medieval, fruto, a su vez, de las pocas excavaciones realizadas en este campo. Este desinterés también queda constatado en el escaso número de comunicaciones que sobre esta temática se presentaron en los distintos Congresos Nacionales de Arqueología celebrados desde 1949 hasta los años finales de la década de los setenta, en los que ni siquiera se contemplaba la dedicación de una sección propia para la etapa medieval.

Aunque a finales del siglo pasado ya se realizaron excavaciones arqueológicas en monumentos medievales de gran importancia, con motivo de restauraciones que en ellos se llevaron a cabo, tales como Santa María de Ripoll, San Juan de las Abadesas o la Alhambra de Granada, no puede considerarse que entonces ya empezase, de una forma sistemática, la práctica de la Arqueología Medieval en nuestro país. No sería hasta el presente siglo cuando este tipo de estudios comenzase a intensificarse, aunque bien es cierto que todavía de una manera muy limitada.

Entre los primeros investigadores cabría citar a don Manuel Gómez-Moreno, de cuya copiosísima obra se pueden señalar algunos trabajos relacionados con aspectos arqueológicos de la Edad Media, aunque casi siempre planteados desde una óptica más bien relacionada con la Historia del Arte, como era costumbre por aquel entonces.

Posiblemente, el periodo histórico medieval que antes despertó un interés arqueológico, reflejado incluso en excavaciones realizadas, fue el visigodo. Ya desde los años 30 empezaron a excavarse diversas necrópolis de esta época, especialmente aquéllas ubicadas en el Valle del Duero. Sin embargo, excepto el mundo funerario, apenas se avanzó en otros campos, como por ejemplo el del hábitat. Entre los primeros arqueólogos que se dedicaron al estudio de esta época cabría señalar a E. Camps Cazorla, J. Martinez Santa Olalla, C. de Mergelina, J. Pérez de Barradas, W. Reinhart, H. Schlunck, B. Taracena, L. Vázquez de Parga, H. Zeiss o G. Nieto Gallo.

El investigador que posteriormente se ha dedicado a un análisis arqueológico más sistemático de esta época, y sobre todo a la transición del mundo tardorromano al visigodo, recogido en una gran cantidad de publicaciones, ha sido Pedro Palol Salellas. Otro gran especialista en este periodo es Luis Caballero Zoreda, fundamentalmente estudioso de la problemática arquitectónica de los edificios religiosos.

Uno de los ámbitos que más llamó la atención de los investigadores, tal vez por la mayor abundancia y espectacularidad de los monumentos conservados, fue el hispanomusulmán. Aquí es inevitable resaltar la gran labor realizada por don Leopoldo Torres Balbás, arquitecto de profesión, que en una gran multitud de estudios, analizó pormenorizadamente el mundo urbano andalusí. lgualmente, para el mundo musulmán, son de destacar personajes como Henri Terrasse, Manuel Ocaña o Félix Hernández. Para una generación más reciente habría que señalar a Guillermo Roselló-Bordoy, Juan Zozaya y Miquel Barceló, éstos posiblemente con una formación arqueológica más estricta, consecuencia de los trabajos de campo que han realizado. Evidentemente son muchos más los investigadores que han analizado arqueológicamente al-Andalus; aquí solamente quedan indicados aquellos que más han destacado, especialmente en esa cierta faceta "pionera" que a muchos de ellos se les puede adjudicar.

El área de los denominados "reinos cristianos", por el contrario, tradicionalmente despertó poco interés arqueológico. Tal vez ello se deba, entre otros factores, a que se trata de zonas en las que la documentación escrita conservada es relativamente abundante, por lo que se contaba con elementos importantes para reconstruir su historia. De ahí también, por ejemplo, que la historia interna de los reinos cristianos se haya conocido mejor que la de al-Andalus. No obstante, no por ello se dejaron de realizar algunos trabajos, muchos de los cuales resultaron de un gran interés por la novedad que suponían y las posibilidades de investigación que abrían. Como ejemplo de uno de estos trabajos cabría señalar las excavaciones arqueológicas que se realizaron en el subsuelo de la catedral de Santiago de Compostela. En el área castellana merece destacarse la labor que llevaron a cabo García Guinea y sobre todo, don Alberto del Castillo, con su estudio de las necrópolis altomedievales, al que se ha considerado como el "padre" de la Arqueología Medieval hispanocristiana, en torno al cual se constituyó un 
grupo de colaboradores que posteriormente llevaron a cabo también una gran labor arqueológica en las zonas del norte peninsular. Para el área catalana son de destacar las excavaciones efectuadas por don Manuel Riu Riu en el ámbito rural, otro de los pioneros de esta actividad.

Este panorama, que aquí sucintamente presentamos, es el que consideramos que refleja la evolución de la Arqueología Medieval en España hasta la década de los sesenta, inclusive. A lo largo de la década siguiente se produjo un incremento considerable en este ámbito de investigación, que se tradujo en un aumento de los yacimientos en proceso de excavación y, por consiguiente, en la producción bibliográfica. A los arqueólogos más veteranos se unieron otros más jóvenes -formados, en la mayor parte de los casos junto a ellos- por lo que la nómina de los arqueólogos medievalistas, hasta entonces bastante reducida, también se incrementó.

Si en todos los campos de investigación los trabajos experimentaron un fuerte impulso, fue sobre todo en el ámbito andalusí en el que más se avanzó, ya que se comenzaron a excavar importantes yacimientos, a la par que se empezaban a sistematizar otros muchos temas (fortificaciones, cerámica,.). En el área cristiana también se intensificaron los trabajos, aunque a un rítmo menor, pero ello supuso un gran avance frente al escaso desarrollo que había tenido hasta entonces. Sin embargo, para la etapa visigoda el proceso fue mucho más lento.

Las causas que podrín haber incidido en este repentino -y en ocasiones, espectacular- impulso de la Arqueología Medieval en nuestro país, son difíciles de explicar. Por un lado, no habría que olvidar la posible repercusión que el desarrollo de la Arqueología, en general, había experimentado hasta entonces. Las etapas prehistóricas, de colonizaciones, romana, etc., estaban siendo bastante estudiadas, aunque no se llegasen a agotar, evidentemente. Sin embargo, era posible que el arqueólogo necesitase nuevos ámbitos en los que intervenir, que estuviesen menos "tocados". De ahí que la Edad Media le ofrecía todo un diversificado campo en el que poder actuar. A ello también habría que añadir el especial interés que por esta etapa histórica, a todos los niveles, se despertó en aquellos años. Igual repercusión pudieron haber tenido las nuevas corrientes teóricas y metodológicas que por entonces también se difundieron y que podían encontrar en la Arqueología Medieval un buen contexto de aplicación.

Además, las fuentes documentales, que eran las que hasta entonces casi en exclusividad se habían empleado para recomponer el pasado medieval, parecía que podían llegar a "agotarse". De ahí que se necesitasen otros recursos con los que proseguir la investigación y que ofreciesen otros frutos distintos o complementarios a los documentos escritos. Y esa vía la podía proporcionar la Arqueología, especialmente para abordar el análisis de muchos temas problemáticos que presentaba la Historia Medieval peninsular. Consideramos que todos estos factores, en mayor o menor medida unos u otros, convergieron en la década de los setenta para animar el panorama arqueológico medieval de España que, de esta manera, recuperó una parte del retraso que llevaba con respecto a otros países europeOS.

La pléyade de jóvenes arqueólogos medievalistas que empezó a surgir, necesitó pronto contar con unos cauces de conexión y de comunicación para coordinar los esfuerzos que se estaban realizando. De ahí que se vió pronto como muy necesaria y conveniente la constitución de una Asociación de Arqueología Medieval que los aglutinase y fuese el cauce a través del cual se expresasen y divulgasen los trabajos en elaboración y los resultados obtenidos, así como cualquier otro tipo de problemas que surgiesen referentes a esta actividad.

De esta manera, $y$ tras un primer encuentro de arqueólogos medievalistas celebrada en el entonces Colegio Universitario de Toledo en el mes de mayo de 1980, dos años después se fundó la Asociación Española de Arqueología Medieval. Consideramos que la fecha tiene su importancia en lo que es la pequeña historia de la Arqueología Medieval española, pues vino a marcar un punto de inflexión en la trayectoria hasta entonces seguida, de manera que se puede hablar de un antes y un después.

Como actividades llevadas a cabo por la citada Asociación merecen destacarse la publicación de un Boletín y la organización de los Congresos Nacionales de Arqueología Medieval. 
Desde el año 1986 se ha empezado a publicar una revista (Boletín de Arqueología Medieval), con periodicidad anual, en la que se recogen artículos de investigación de carácter diverso, así como información sobre excavaciones que se están llevando a cabo. Este Boletín es la primera, y hasta ahora la única, publicación española dedicada exclusivamente a la difusión de temas de Arqueología Medieval, y se ha convertido en el equivalente español de otras revistas de carácter similar publicadas desde hace años en otros países europeos (Medieval Archaeology, Archologie Médiévale, Archeologia Medievale).

Por otro lado, uno de los grandes logros de esta Asociación ha sido la organización de los Congresos Nacionales de Arqueología Medieval, de los que ya se han celebrado 4 hasta la fecha (Huesca 1985, Madrid 1987, Oviedo 1989 y Alicante 1993) y que han sido también los primeros que con esta temática monográfica se han celebrado en nuestro país, todos ellos además con un gran éxito de participantes y de comunicantes, como se refleja en las correspondientes actas que se han editado.

Sin embargo, a pesar de este incuestionable desarrollo, todavía la enseñanza de la Arqueología Medieval en los niveles superiores apenas se tiene en consideración y son pocas las Universidades en las que queda recogida como una asignatura individualizada en los planes de estudios o con dotación de plazas específicas. Suele ser la labor personal de algunos profesores, especialmente interesados en estos temas, la que ha impulsado su actividad en algunos departamentos. Y ello se ha reflejado en la programación de excavaciones, en la elaboración de tesis doctorales, en la organización de reuniones de carácter científico y en la dedicación de sesiones específicas en las revistas que editan (Acta Historica et Archaeologica Mediaevalia de la Universidad Central de Barcelona o Estudios de Historia y Arqueología Medievales de la Universidad de Cádiz).

Si, evidentemente, la labor paciente y emprendedora de muchos investigadores ha supuesto que se haya avanzado bastante en los últimos años y que la Arqueología Medieval esté teniendo cada vez más un mayor reconocimiento, es todavía mucha la labor que queda por realizar.

\section{TEMAS ESTUDIADOS}

El progresivo incremento de la actividad investigadora en el campo de la Arqueología Medieval en nuestro país, ha llevado a elaborar algunos ensayos de sistematización de la misma, para establecer lo que podríamos denominar como "el estado de la cuestión".

La primera sistematización se realizó por Manuel Ríu en el año 1977. Posteriormente, en 1985, con motivo del I Congreso de Arqueología Medieval Española, este mismo investigador presentó una ponencia en la que se actualizaba el trabajo anterior, con todos los estudios que a lo largo de los 10 años transcurridos se habían publicado, aunque limitándose al campo de los reinos cristianos (Riu, 1986). En este mismo Congreso, Guillermo Roselló-Bordoy presentó otra ponencia analizando la situación de la arqueología andalusí (Roselló 1986). Con ambos trabajos se obtiene un amplio panorama del estado de la Arqueología Medieval española en dichas áreas, aunque todavía falta por sistematizar lo concerniente a la etapa visigoda.

La situación de la Arqueología Medieval española, en cuanto a los temas que con más profusión se están estudiando, puede establecerse, en cierta medida, a partir del análisis del contenido de las actas de los Congresos Nacionales de Arqueología Medieval que se han celebrado, pues en ellas están recogidos los resultados de los trabajos terminados o en proceso de elaboración. Por lo cual, nos ofrecen esa panorámica de conjunto de las investigaciones más recientes, y por ello el estado actual más aproximado, que se puede complementar con otras muy diversas publicaciones.

No pretendemos analizar exhaustivamente todas las ponencias y comunicaciones en los Congresos, ni otros estudios publicados en otros contextos, ni tampoco establecer una clasificación temática de los mismos, sino simplemente señalar las líneas generales por las que parece encauzarse la investigación, para constatar posibles preferencias por ciertos temas específicos. Por ello, vamos a omitir toda referencia a autores y títulos, pues en tal caso se excederían ampliamente los propósitos de esta comunicación. En primer lugar, para abordar este análisis vamos a seguir el mismo orden normalmente establecido en las distintas secciones de los 
Congresos: metodología, etapa visigoda, al-Andalus y reinos cristianos.

Bajo el epígrafe de "Metodología" se suelen recoger una gran variedad de estudios, sin que tengan una intención de exponer planteamientos de carácter estrictamente metodológico (ni teórico ni práctico). Tratan temas muy heterogéneos, siempre interesantes, muchos de los cuales podrían perfectamente incluirse en la correspondiente sección histórica de la que tratan. Parece existir una cierta preocupación por concretar la situación de la Arqueología Medieval en ciertas regiones o provincias (País Vasco, Asturias, Cataluña, Castilla-La Mancha, Avila, etc.) y por elaborar cartas arqueológicas (Cataluña, Asturias, País Vasco, etc.).

De la misma manera, son varios los estudios dedicados al análisis metodológico y terminológico de la arquitectura militar. Igualmente, se constata un gran interés por la problemática surgida en torno a la restauración de monumentos o de intervenciones de consolidación en yacimientos medievales. Los estudios faunísticos y su interpretación son objeto también de una atención que va en aumento. No así, hasta el momento, los relacionados con los análisis antropológicos, aunque bien es cierto que muchos resultados de este tipo se encuentran recogidos en las correspondientes memorias de excavaciones y no como monografías aisladas. También el estudio de los graffiti parecen despertar cierta curiosidad. Algo menor es la de los análisis numismáticos, tanto en el campo cristiano como en el musulmán, así como el de los temas de carácter tecnológico y los relacionados con las técnicas de excavación.

En cuanto al estudio de la arqueología de época visigoda, continúan despertando interés prioritario los temas relacionados con el mundo funerario (análisis de iglesias y eremitorios). También se constata una preocupación por el estudio de poblados específicos y por la dispersión de las fortificaciones de esta época, temas de gran interés que hasta ahora apenas se habían tenido en consideración. La cerámica de esta época, en gran medida desconocida, también está siendo objeto de estudio. Igualmente se siguen analizando materiales individuales (piezas arquitectónicas, de orfebrería, etc.). Lo novedoso es que se empieza a constatar una preocupación por un aspecto de gran interés, hasta el presente apenas resaltado: la pervivencia de elementos de época visigoda tras la llegada de los musulmanes, lo que abre un campo interesantísimo de investigación -y clave en muchos aspectos- en la esfera histórico-arqueológica.

Por lo que respecta a la arqueología andalusí, por su número destacan los estudios relativos al análisis de la cerámica (hallazgos aislados, conjuntos de yacimientos concretos, tipologías, decoraciones, etc.). Ello no es sorprendente si tenemos en cuenta la abundancia de este material en cualquier excavación y la necesidad de su estudio -siquiera sea como simple elemento cronológico de referencia-, al que hasta épocas recientes apenas se había tenido en consideración. También se observa un interés, lógico, por la dispersión del poblamiento de alAndalus -rural y urbano- tanto de regiones extensas (Las Alpujarras, zona levantina, etc.) como de lugares más concretos en los que se realizan excavaciones, bien de una manera prolongada (Calatrava la Vieja, Vascos, Alarcos, Pechina, etc.) o de una forma esporádica con motivo de intervenciones de urgencia (Sevilla, Lérida, Valencia, etc.). Especial interés están despertando los estudios relacionados con el análisis de las estructuras urbanísticas, y más concretamente sobre el ámbito doméstico.

También numerosos son los estudios sobre las construcciones militares, analizadas tanto desde el punto de vista de sistemas defensivos organizados, como de simples enclaves individuales (castillos, torres, atalayas, alcazabas, murallas, etc.). Asimismo, algunos conjuntos monumentales de excepcional envergadura, como Madínat al-Zahrā o la Alhambra, siguen siendo objeto de análisis.

Existe un interés creciente por los temas relacionados con los sistemas hidráulicos, su configuración técnica y sus diversos aprovechamientos (regadíos, abastecimiento de agua a lugares habitados, almacenamiento, etc.).

El ámbito funerario, hasta ahora apenas tenido en consideración arqueológica, cada vez está despertando más interés, al ser localizados y excavados algunos cementerios. También algunas mezquitas y otros edificios significativos (baños) son objeto de estudio, aunque en menor proporción. Por último, existen estudios de carácter más heterogéneo, 
como los que analizan piezas de material diverso (marfil, vidrio, etc.).

En cuanto a la arqueología de los reinos cristianos, la mayor abundancia de estudios se centra también en la cerámica, y en ellos se analizan conjuntos procedentes de zonas y etapas cronológicas muy diversas. Les siguen los estudios relacionados con las construcciones militares, que se analizan bien de una manera individualizada (castillos, torres, murallas, etc.) o bien formando parte de sistemas defensivos de gran amplitud territorial.

Igualmente numerosos son los trabajos referentes al análisis del poblamiento, tanto desde el punto de vista de su dispersión, como de yacimientos que han sido objeto de excavación. Por lo que respecta al ámbito religioso, han sido varias las iglesias rurales que se han excavado, así como algunos eremitorios rupestres y monasterios. En cuanto al mundo funerario, también han sido varios los cementerios excavados; especial interés despierta el análisis de las estelas encontradas en muchos de ellos.

Si ahora prescindimos de cada una de las etapas o fases históricas y abarcamos la Arqueología Medieval en su conjunto, por lo que respecta a los temas que más se están estudiando parece observarse una cierta prioridad, al menos numérica, por el de la cerámica, tanto en la arqueología andalusí como en la de los reinos cristianos. Esta aparente preferencia por el estudio de la cerámica tiene, en parte, su explicación lógica, por tratarse de un material muy abundante, que en mayor o menor grado de conservación aparece en todos los yacimientos. Ello ha supuesto que los arqueólogos se han ido encontrando con una gran abundancia de fragmentos y piezas cerámicas, muchas de ellas desconocidas, ya que anteriormente no habían sido estudiadas. Por lo cual, han tenido que empezar a sistematizar todo ese material -dado, además, el gran valor referencial cronológico que la cerámica tiene- y de ahí la gran cantidad de estudios -de extensión y calidad muy variable- que se vienen publicando.

Sin embargo, conviene señalar que, a pesar del importante aporte que, en conjunto, todos esos estudios suponen, la mayor parte de los mismos están planteados con unos criterios estrictamente arqueológicos, es decir, con descripciones de las piezas (tipologías, calidad de las pastas, colores, decoraciones, etc.) y establecimiento de paralelos con otras similares. Sin embargo, con ser esto importante y fundamental, faltan ensayos de sistematización -aunque se han efectuado para áreas concretas- y estudios para encuadrar a la cerámica en los contextos históricos en los que surge, de cara a conseguir una información más extensa que la exclusivamente taxonómica. Es de suponer que en los próximos años disminuirán los estudios sobre cerámica y su análisis se someterá a otros criterios metodológicos. Así, se analizarán los focos de producción, los sistemas de distribución, las influencias de las modas, el carácter simbólico de la decoración, etc.

Otro de los temas bastante estudiados, tanto en la arqueología andalusí como en la cristiana, es el referente a las fortificaciones o construcciones militares (castillos, torres, atalayas, alcazabas, murallas, etc.). Muchos de ellos están elaborados con unos criterios puramente descriptivos, aunque otros presentan los resultados de excavaciones que en los mismos se han realizado. Estos estudios son de un gran interés -dado también el especial significado sociopolítico de las fortificaciones en la Edad Mediaaunque la mayor parte de los mismos se limitan a analizar -arquitectónica o arqueológicamente- alguna fortificación concreta, aislada de su contexto. Aunque ya se van elaborando trabajos en los que se estudian redes de sistemas defensivos, es conveniente que éstos se orienten cada vez más por la vía de una arqueología espacial, por la cual encuadrar las fortificaciones en el contexto estratégicomilitar en el que surgen, a la par que analizar la función política, social y económica que desempeñan en cuanto a la organización del territorio- y su relación con otras fortificaciones con las que, en definitiva, forman un sistema defensivo organizado. Aquí, por consiguiente, se abre un importante campo para futuras investigaciones.

Otro tema que también despierta gran interés en la Arqueología Medieval española es el relacionado con la excavación de núcleos de población, muy especialmente a nivel rural (salvo para algunos casos referentes a al-Andalus, puede decirse que no existe todavía una arqueología urbana sistemática). Son bastantes los puntos de hábitat que se han - o están siendo- excavados, con resultados muy interesantes de cara a obtener una abundante e 
inédita información sobre los individuos que los poblaron. No obstante, muchos de estos estudios siguen unos planteamientos estrictamente descriptivos a la hora de presentar el proceso de excavación, así como los materiales encontrados. Se hace cada vez más necesario poner en práctica nuevos sistemas metodológicos, que nos puedan proporcionar una información más variada y compleja, aunque bien es cierto que no exentos de algún riesgo de convicción en cuanto a su interpretación: modos de vida, alimentación (mediante análisis de los restos de fauna que se recogen), actividad económica, organización social, etc. Es evidente que para conseguir todos estos datos es necesario un trabajo interdisciplinar, lo que implica que el arqueólogo cada vez menos puede actuar de una manera aislada.

De la misma manera, los núcleos de población no se pueden tampoco analizar de una forma individualizada, limitados exclusivamente a lo que fue la superficie propiamente habitada. En torno a los mismos el espacio se organizó por y para ellos, mediante una actuación antrópica que es conveniente captar. De ahí el interés por reconstruir los límites de ese espacio -especialmente mediante prospecciones- para obtener un conocimiento más exhaustivo de la organización territorial.

Igualmente, los lugares habitados no permanecían aislados sino que existía una intercomunicación entre ellos, en la que las vías de comunicación (los caminos) ejercían un papel fundamental. De ahí también el interés por conocer la red viaria, tema éste que hasta ahora apenas se ha tenido en consideración.

El mundo funerario -la denominada arqueología de la muerte- también ha sido otro de los temas en los que se ha trabajado bastante, aunque los estudios casi siempre se han limitado a la excavación de necrópolis y al análisis descriptivo de los resultados arqueológicos. Es de señalar que en este campo se ha trabajado más en la zona cristiana que en la andalusí (para la etapa visigoda ya hemos señalado anteriormente cómo la mayor parte de las investigaciones tradicionalmente se han centrado en la excavación de necrópolis). Aunque ya se han elaborado estudios de sistematización, éstos no pasan de planteamientos puramente tipológicos, por lo que se hace necesaria una mayor profundiza- ción en toda la problemática funeraria (actitudes ante la muerte, ritos funerarios, etc.), que debe de ir acompañada de los correspondientes análisis antropológicos que pueden proporcionar una información complementaria de gran riqueza (sexo de los individuos, enfermedades, medias de vida, comportamientos sociales, etc.). El estudio de los cementerios hay que encuadrarlo también en ese proceso de organización espacial señalado con anterioridad.

Lo que podríamos denominar como el ámbito religioso ha sido un tema algo menos estudiado, aunque se han excavado edificios de culto, tanto en la etapa visigoda (iglesias, eremitorios), como en los reinos cristianos (iglesias, ermitas), así como en alAndalus (mezquitas). Sin embargo, estos estudios no han sido muy numerosos, teniendo en cuenta que gran parte de la arquitectura religiosa medieval se conserva y está en uso, por lo que realizar excavaciones resulta muy complejo. De ahí que la "arqueología de la religión" se haya enfocado, sobre todo, con unos criterios descriptivos, más en consonancia con los planteamientos de la Historia del Arte. No obstante, aquí se abre un campo de investigación en verdad interesantísimo, sobre todo para los primeros tiempos medievales, asociados a la difusión del cristianismo y su adaptación a una religiosidad pagana, e igualmente para el momento del establecimiento de los musulmanes y su proceso de organización religiosa en convivencia con otros cultos. La comprobación arqueológica de esos cambios puede marcar una línea de investigación muy interesante.

Hasta aquí hemos expuesto algunas consideraciones sobre los temas que mayor interés han despertado entre los arqueólogos medievalistas. Sin embargo, no son los únicos que se han investigado, aunque sí son aquellos que implican una mayor envergadura por la amplitud de los problemas que pueden abarcar. Los trabajos sobre temas más específicos y concretos suelen ser también numerosos y heterogéneos. Así, por ejemplo, son frecuentes las publicaciones de piezas de materiales diversos (hueso, metal, vidrio, cerámica, piezas escultóricas, etc.) que son interesantes, aunque las piezas pueden resultar un tanto descontextualizadas, por lo que solamente adquieren una utilidad para ser consideradas como elementos de referencia cronológica o tipológica. 
Los estudios sobre materiales numismáticos no son muy abundantes -tal vez en consonancia con una escasez de hallazgos de esta naturaleza-, aunque no hay que olvidar que el análisis de monedas no tiene una componente exclusivamente arqueológica.

Es de destacar también el escaso interés que ha despertado la arqueología asociada a las minorías sociorreligiosas (mozárabes, judíos y mudéjares). Aunque bien es cierto que éstas no han generado unos elementos materiales específicos (normalmente utilizaban los de la sociedad en la que se encontraban conviviendo), sin embargo, su propia diferenciación religiosa originaba algunos matices que pueden tener su constatación arqueológica (lugares de culto, de enterramiento, etc.). Aquí también hay, por tanto, otro campo en el que intensificar la investigación.

Por último, señalar que el progresivo desarrollo que está teniendo la Arqueología Medieval en nuestro país está repercutiendo en la necesidad de empezar a elaborar trabajos de síntesis o de sistematización. Así, los que se orientan por la confección de cartas arqueológicas o el análisis del estado de la Arqueología Medieval en ciertas comunidades o provincias. Igualmente, los que intentan sistematizar determinados campos ya bastante investigados: tipologías cerámicas, de enterramientos, de armamento, de fortificaciones, etc.

En general, y si nos atenemos al número de publicaciones, parece que la arqueología andalusí es la que cuenta con una mayor cantidad de investigadores. Ello tal vez se deba a la gran extensión territorial y cronológica que llegó a abarcar la presencia musulmana en nuestro suelo, y de ahí la gran abundancia de yacimientos, muchos de ellos de una gran espectacularidad, que han despertado el interés por su investigación. La necesidad de un conocimiento más profundo de dicha etapa, todavía con muchas lagunas en el campo histórico, ha podido influir en ese interés, aunque en el mismo se constata una desproporción acusada en cuanto a las distintas fases políticas de al-Andalus.

Así, los primeros momentos del asentamiento de los musulmanes (la denominada arqueológicamente como etapa paleoandalusi) apenas han sido analizados y algo similar ocurre con la etapa emiral.
La mayor parte de los estudios arqueológicos se han centrado en las etapas del Califato y de las Taifas, mientras que el siglo XII (almorávides y almohades) todavía está bastante desconocido y el reino de Granada está despertando un interés creciente. Como se puede constatar, esta situación tiene un evidente paralelismo con lo que sucede en el campo de la investigación propiamente histórica de al-Andalus.

En cuanto a los reinos cristianos, también están siendo objeto de numerosos estudios arqueológicos y a ello igualmente ha podido contribuir la necesidad de complementar una información imposible de precisar, muchas veces, por vía documental. Muchas de las grandes áreas de investigación, abordadas mediante la utilización de una documentación escrita, requieren ser complementadas con unos datos que solamente se pueden obtener por una vía arqueológica.

De ahí el progresivo interés por propiciar este tipo de estudios, entre los que se observa una preferencia por la época altomedieval, lo cual puede explicarse por una necesidad de conseguir nuevos datos históricos para una etapa en la que la documentación escrita no es excesivamente abundante, y de ahí el recurrir a la aplicación de otros sistemas metodológicos de investigación. Por motivos contrarios, la Baja Edad Media está siendo mucho menos estudiada arqueológicamente, pues la documentación, mucho más rica y abundante, aporta una información más completa, aunque ello no signifique que haya que desechar la utilización de la arqueología para este periodo.

La etapa visigoda es la que menor interés arqueológico despierta, en comparación con las otras dos. Si inicialmente fue la que más se investigó, incluso casi con una cierta exclusividad, posteriormente aquél se orientó hacia los campos andalusí y cristiano, aunque ello no suponga que el periodo visigodo carezca ya de interés; lo sigue teniendo, y mucho, pues es una etapa clave para comprender gran parte de la problemática arqueológica de la etapa siguiente, al menos en sus primeros momentos. Lo que ocurre es que las áreas de investigación apenas han evolucionado -se siguen estudiando, de preferencia, necrópolis- y de ahí esa apariencia de cierto estancamiento que puede ofrecer, aunque se siga trabajando y excavando bastante. 
Este es el panorama, sucintamente expuesto, que en estos momentos parece presentar la Arqueología Medieval en España. Es evidente que en los últimos años se ha avanzado mucho, al menos en el campo de la investigación, recuperando gran parte del retraso que en este ámbito se llevaba. La década de los ochenta ha sido muy fructífera a muchos niveles (reuniones científicas, publicaciones, excavaciones, etc.) pero, ien qué punto nos encontramos? ¿Sigue el avance? ¿A qué ritmo? Dentro de unos años, con la perspectiva del paso del tiempo y los resultados obtenidos, podremos contestar a estas preguntas y así seguir el pulso de la trayectoria de la investigación arqueológica de nuestra Edad Media.

\section{BIBLIOGRAFÍA}

BARCELÓ, Miquel y otros (1988): La Arqueología Medieval. En las afueras del "medievalismo". Barcelona, 1988.

RIU, Manuel (1977): "La Arqueología Medieval en España", en Manual de Arqueología Medieval de Michel de BOUARD. Barcelona, pp. 375-490.

RIU, Manuel (1986): "Estado actual de la Arqueología Medieval en los reinos cristianos peninsulares", en Actas del I Congreso de Arqueología Medieval Española, vol. IV. Zaragoza, pp. 425-472.

ROSELLÓ-BORDOY, Guillermo (1986): "Islam andalusí e investigación arqueológica. Estado de la cuestión", en Actas del I Congreso de Arqueología Medieval Española, vol. III. Zaragoza, pp. 7-24. 